Published in final edited form as:

Am J Transplant. 2019 April ; 19(4): 1187-1194. doi:10.1111/ajt.15150.

\title{
In-hospital and 90-day outcomes after total pancreatectomy with islet autotransplantation for pediatric chronic and acute recurrent pancreatitis
}

\author{
Meera Kotagal $^{1}$, Joyce Slusher ${ }^{1}$, Syed Ahmad ${ }^{2,3}$, Lori A. Aronson ${ }^{4,5}$, John Brunner ${ }^{2}$, Ranjit \\ Chima $^{5,6}$, Deborah A. Elder ${ }^{5,7}$, Kenneth R. Goldschneider ${ }^{8}$, Lindsey Hornung ${ }^{9}$, Tom K. \\ Lin $^{5,10}$, Stephen M. Ogg ${ }^{1}$, Joseph J. Palermo ${ }^{5,10}$, Kristin Rich ${ }^{5,11}$, John Rose ${ }^{8}$, Stephen \\ Sekoulopoulos ${ }^{1}$, Alexandra Szabovaa ${ }^{8}$, Maisam Abu-EI-Haija ${ }^{5,10}$, Jaimie D. Nathan ${ }^{1,12}$ \\ ${ }^{1}$ Division of Pediatric General and Thoracic Surgery, Cincinnati Children's Hospital Medical \\ Center, Cincinnati, Ohio \\ ${ }^{2}$ Division of Surgical Oncology, Pancreas Disease Center, University of Cincinnati Medical Center, \\ Cincinnati, Ohio \\ ${ }^{3}$ Pancreas Disease Center, University of Cincinnati Medical Center, Cincinnati, Ohio \\ ${ }^{4}$ Department of Anesthesiology, Cincinnati Children's Hospital Medical Center, Cincinnati, Ohio \\ ${ }^{5}$ Department of Pediatrics, University of Cincinnati College of Medicine, Cincinnati, Ohio \\ ${ }^{6}$ Division of Critical Care Medicine, Cincinnati Children's Hospital Medical Center, Cincinnati, Ohio \\ ${ }^{7}$ Division of Endocrinology, Cincinnati Children's Hospital Medical Center, Cincinnati, Ohio \\ ${ }^{8}$ Pain Management Center, Department of Anesthesiology, Cincinnati Children's Hospital Medical \\ Center, Cincinnati, Ohio \\ ${ }^{9}$ Division of Biostatistics and Epidemiology, Cincinnati Children's Hospital Medical Center, \\ Cincinnati, Ohio \\ ${ }^{10}$ Division of Pediatric Gastroenterology, Cincinnati Children's Hospital Medical Center, Cincinnati, \\ Ohio \\ ${ }^{11}$ Division of Behavioral Medicine and Clinical Psychology, Cincinnati Children's Hospital Medical \\ Center, Cincinnati, Ohio \\ ${ }^{12}$ Department of Surgery, University of Cincinnati College of Medicine, Cincinnati, Ohio
}

\author{
Abstract \\ Correspondence: Jaimie D. Nathan, jaimie.nathan@cchmc.org. \\ Jaimie D. Nathan and Maisam Abu-El-Haija contributed equally to the manuscript as co-senior authors. \\ Meeting Presentations: This work was presented as an abstract at the American Pediatric Surgical Association (APSA) Meeting, \\ Palm Springs, CA, May 2018. \\ DISCLOSURE \\ The authors of this manuscript have conflicts of interest to disclose as described by the American Journal of Transplantation. Tom K. \\ Lin, MD: Medtronic, payment for lecture on capsule endoscopy. John K. Rose, MD: Data Safety Monitoring Board for Grunethal, \\ pediatric Tapentadol trials. The other authors have no conflicts of interest to disclose.
}


Total pancreatectomy with islet autotransplantation (TPIAT) is used to treat debilitating chronic pancreatitis (CP) and acute recurrent pancreatitis (ARP) that has failed medical and endoscopic therapy. We performed a retrospective review of TPIAT patients at a free-standing children's hospital to evaluate perioperative outcomes. Twenty patients (median age 13,65\% female) underwent TPIAT (2015 through 2017). Of the 20 patients, 95\% had CP and 1 patient (5\%) had ARP alone. Seventy-five percent of the patients had a pancreatitis-associated genetic mutation; $40 \%$ had pancreas divisum. The median surgical time was 757 (IQR 657 to 835) minutes. Median islet equivalents per $\mathrm{kg}$ of body weight (IEQ/kg) were 6404 (IQR 5018 to 7554). At 90 days postoperatively vs preoperatively, significantly fewer patients were receiving parenteral nutrition ( $0 \%$ vs $25 \%, P=.006$ ) and opioids ( $45 \%$ vs $75 \%, P=.01$ ). Short Form 36-Item Health Survey (SF-36) physical health module scores and total scores improved (34.0 preoperatively vs 54.6 at 90 days, $P=.008$, and 47.1 vs $65.3, P=.007$, respectively); SF-10 physical health scores also improved (13.4 vs 33.1, $P=.02$ ). Insulin requirement decreased from $0.5 \mathrm{unit} / \mathrm{kg} / \mathrm{day}$ to $0.4 \mathrm{unit} / \mathrm{kg} / \mathrm{day}$ between discharge and 90 days $(P=.02)$. TPIAT is an effective option when debilitating disease persists despite maximal medical and endoscopic therapy. Opioid, parenteral nutrition, and exogenous insulin use can successfully be weaned within 90 days after TPIAT, with gains in health-related quality of life.

\section{Keywords}

autotransplantation; clinical research/practice; health services and outcomes research; insulin/Cpeptide; islet transplantation; nutrition; pediatrics; quality of life (QOL)

\section{1 | INTRODUCTION}

Chronic pancreatitis (CP) and acute recurrent pancreatitis (ARP) cause significant morbidity for both adult and pediatric patients, with recurrent inflammation resulting in irreversible parenchymal damage (eg, fibrosis, atrophy) and significant symptomatology. While the overall incidence of CP is approximately $0.5 \%$ in the United States, ${ }^{1,2}$ the annual health care expenditure of $\$ 2.6$ billion is a significant financial burden. ${ }^{3}$ The incidence of acute pancreatitis (AP) in children has been estimated between 3.6 and 13.2 cases per 100 000 children, with a notable increase during the past 2 decades. ${ }^{4-7}$ This observed rise in pediatric AP has mirrored the increase in $\mathrm{CP}$ admissions during the past decade. ${ }^{8}$ Similar to adult disease patterns, pediatric $\mathrm{CP}$ may follow a course of progressive pain severity and opioid dependence, with the progression of disease to exocrine and endocrine insufficiency. ${ }^{9}$ Children with CP have health-related quality of life (HRQOL) scores that are 1 to 2 SDs below the average, consistent with HRQOL scores for cancer, end-stage renal disease, and rheumatologic diseases in children, ${ }^{10,11}$ indicating the profound effect of the disease on their daily functioning, with recurrent hospitalizations, school absences, and impaired interactions with family and peers. ${ }^{12} \mathrm{CP}$ results in malnutrition and feeding challenges, further highlighting the need to recognize it and intervene promptly. ${ }^{13}$

Total pancreatectomy with islet autotransplantation (TPIAT) has been performed in adult populations for $>4$ decades, having first been described in $1977 .{ }^{14}$ In adult populations, post-TPIAT patients have demonstrated significant decreases in opioid requirements and 
improved quality of life. ${ }^{15-18}$ The first pediatric TPIAT was performed in $1989,{ }^{19}$ yet data on TPIAT in pediatric populations remain limited. ${ }^{20}$ In this study, we report post-TPIAT outcomes from a single free-standing pediatric academic medical center, focusing on immediate preoperative, in-hospital, and 90-day postoperative outcomes, including opioid, parenteral nutrition (PN), and exogenous insulin use; $\mathrm{C}$-peptide levels as a marker of $\beta$-cell function; and HRQOL. Additionally, we are the first to report on the early benefits of TPIAT on supplemental nutritional needs in children.

\section{2 | MATERIALS AND METHODS}

A retrospective review was performed of pediatric patients who underwent TPIAT at Cincinnati Children's Hospital Medical Center (CCHMC) between April 2015 and August 2017. Twenty consecutive patients were included in the review. Demographic information, preoperative characteristics (including genetic risk factors, nutritional supplementation, and opioid requirements), in-hospital course (including operative duration, length of stay, and islet yields), and 90-day postoperative outcomes (including complications, changes in opioid and nutritional supplementation, and HRQOL) were abstracted from the clinical record. The study was approved by the Institutional Review Board at CCHMC.

\section{1 | Patient selection}

Potential patients with $\mathrm{CP}$ and ARP were evaluated by a multidisciplinary team that included pediatric subspecialists in the following areas: transplant surgery, gastroenterology, pain management, endocrinology, and psychology. The appropriateness of recommending a TPIAT to a patient was based on a comprehensive review and assessment of the child's prior clinical course, response to treatments and interventions, and their current level of impairment of quality of life and daily functioning. $\mathrm{CP}$ and ARP in the pediatric population were defined as published by the International Study Group of Pediatric Pancreatitis: In Search for a Cure (INSPPIRE) consortium. ${ }^{21}$ Exocrine pancreatic insufficiency was defined as fecal elastase level $<200 \mu \mathrm{g} / \mathrm{g}$ stool or an endoscopic pancreatic function test consistent with exocrine insufficiency. Patients and their families underwent thorough preoperative counseling regarding the nature of the operation, as well as the potential risks and the benefits. In addition, they participated in extensive education and preparation with regard to pain management (including opioid use) before, during, and after TPIAT. Given the inclusion of splenectomy as part of the surgical intervention, all patients underwent a preoperative assessment of their vaccination status for encapsulated organisms; if determined to be deficient, the appropriate vaccinations were administered before surgery.

\section{$2.2 \mid$ Operative procedure}

TPIAT included resection of the pancreas, duodenum (pylorus pre-serving), gallbladder, spleen, and appendix. Pancreatic blood supply was dissected and preserved until shortly before removal of the pancreas to minimize ischemia time for the islets. Islet isolation was performed via mechanical and enzymatic degradation as previously described. ${ }^{22}$ The processing of juvenile pancreata for autologous islet transplant was performed through developmental therapeutics in cooperation with University of Cincinnati, Cellular Therapies. Immediately after total pancreatectomy, the pancreas was taken to a sterile field in the 
operating room, where it was flushed with cold University of Wisconsin solution, trimmed, and packaged in fresh cold preservation solution for immediate transport to the islet processing facility. The pancreas was perfused with Liberase MTF (Roche, Indianapolis, IN) and digested by using the Ricordi Isolator III (Biorep Technologies, Miami, FL). Processed cells were not purified. The cell volume was suspended in serum albumin with 70 units/kg heparin, packaged, and immediately transported to the operating room for fresh infusion. Isolated islets were infused into the portal circulation via cannulation of the splenic vein stump. During infusion, mean arterial pressure, portal venous pressure, central venous pressure, and blood glucose were monitored. Islet infusion was paused if there was a significant rise in portal venous pressure from preinfusion measurements $(>20 \mathrm{~mm} \mathrm{Hg}$ change from starting pressure or absolute value $>25 \mathrm{~mm} \mathrm{Hg}$ ). Dextran infusion was initiated before islet transplant to reduce the instant blood-mediated inflammatory response, ${ }^{23}$ and patients were maintained on an insulin infusion for glycemic control (goal blood glucose level of 80 to $120 \mathrm{mg} / \mathrm{dL}$ ) throughout the operation to maximize survival and engraftment of the islets. Biliary reconstruction was performed via Roux-en-Y choledochojejunostomy or hepaticojejunostomy, and alimentary tract reconstruction was performed via Roux-en-Y duodenojejunostomy. All patients had a gastrojejunostomy tube placed at the time of surgery to allow for early initiation of distal enteral feeds postoperatively.

\subsection{Postoperative care}

All patients were admitted to the intensive care unit (ICU) postoperatively for a standard 7-day ICU stay for administration and monitoring of insulin and heparin infusions. Abdominal ultrasound was performed immediately postoperatively to evaluate for portal vein thrombosis. Blood glucose levels were closely monitored with hourly blood glucose checks and a subcutaneous continuous glucose monitoring system. Insulin infusion was titrated to keep blood glucose levels in the range of 80 to $120 \mathrm{mg} / \mathrm{dL}$ by using a standardized guideline. Continuous glucose monitoring via the use of a Dexcom G4 Platinum Professional monitor (Dexcom, San Diego, CA) was used throughout the hospital stay to allow for early identification of deviation in blood glucose. Patients were maintained on a low-dose heparin infusion (10 units $/ \mathrm{kg} / \mathrm{hr}$ ) for 7 days, before transition to low-molecular-weight heparin subcutaneous prophylaxis. Patients were subsequently transitioned from insulin infusion to insulin pumps and underwent extensive teaching for the maintenance of glycemic control postoperatively. Patients were managed by the acute pain service intraoperatively and postoperatively. Perioperative pain management included extensive use of nonopioid analgesics beginning in the operating room and continuing into the ICU stay. All patients were seen in close follow-up with surgery, gastroenterology, pain management, and endocrine providers after hospital discharge. All patients underwent standard mixed meal testing at 90 days postoperatively to evaluate islet function. ${ }^{24}$

\subsection{Health-related quality of life}

All patients 12 years and older were asked to complete the Short Form 36-Item Health Survey (SF-36), and parents of all patients $<12$ years of age were asked to complete the Short Form 10-Item Health Survey (SF-10) Health Survey for Children. The SF-36 is a 36-item, patient-reported survey of patient health that includes 8 modules composing a 
physical health score and a mental health score. ${ }^{25}$ The SF-10 is a parent-completed survey of patient health and includes physical and psychosocial component scores. ${ }^{26}$

\section{5 | Statistical analysis}

Data were analyzed by using SAS, version 9.4 (SAS Institute, Cary, NC). Due to sample sizes, continuous data were summarized as median values (IQR: 25th-75th percentiles), while categorical data were summarized as frequency counts and percentages. Exact binomial tests were used to compare proportions, and Wilcoxon signed rank tests were used to compare continuous variables preoperatively and 90 days postoperatively. A value of $P<.05$ was considered statistically significant.

\section{3 | RESULTS}

Twenty patients underwent TPIAT between April 2015 and August 2017. Clinical and demographic characteristics of the cohort are described in Table 1. Indication for TPIAT was CP in $95 \%$ of patients, whereas 1 patient had debilitating ARP alone without meeting diagnostic criteria for CP. Within this cohort, $75 \%$ had an identified genetic risk factor for their pancreatitis, and $45 \%$ of patients had an anatomic abnormality, either pancreas divisum or annular pancreas. Seventy-five percent of patients had used opioid medications for management of chronic pain in the year before surgery, and 25\% were receiving partial or full parenteral nutritional support, with an additional three patients requiring enteral feeding supplementation due to failure to thrive and/or intolerance of oral feeds. Sixty percent of patients had preoperative exocrine insufficiency, and 15\% had preoperative endocrine insufficiency with diabetes. Patients undergoing TPIAT had severe debilitating disease with a median of 4.5 hospitalizations in the preceding year.

\section{1| Operative details}

Median operative duration was 757.5 (IQR: 657.5 to 835.5) minutes. Eighty-five percent of patients had all isolated islets infused into the liver via portal vein via cannulation of the splenic vein stump. Three patients had to have portal vein infusion stopped for persistently elevated portal pressures, with the remainder of those islets infused into the peritoneal cavity. The median islet equivalents transplanted per $\mathrm{kg}$ body weight (IEQ/kg) was 6404 (IQR: 5018 to 7554; Table 2).

\section{2 | In-hospital and 90-day outcomes}

Median length of stay was 26 (IQR: 23 to 31 ) days, with a median ICU length of stay of 10 (IQR: 9 to 11) days. Six patients experienced postoperative complications during their initial hospitalization, 5 of which were fluid collections requiring percutaneous drainage. One patient required immediate reexploration for bleeding due to omental venous bleeding related to suction trauma from an internal drain. One additional patient developed an adhesive bowel obstruction necessitating exploration after initial hospital discharge but within the 90 days after surgery (Table 3). At 90 days, all patients were off PN, and 70\% were tolerating full nutrition via mouth with $30 \%$ still requiring enteral supplementation via jejunal tube feeds. The difference in PN use between the preoperative and postoperative periods was statistically significant $(P=.006$, Figure 1$)$. Patients were 
significantly less likely to be using opioids at 90 days postoperatively (45\%), compared with $75 \%$ preoperatively ( $P=.01$, Figure 2$)$. As a measure of opioid use, among patients who were taking opioids preoperatively, the median use was 20 morphine equivalents/day preoperatively, which decreased at 90 days to a median of 7.5 morphine equivalents/day. This difference was not statistically significant $(P=.16)$. Median stimulated C-peptide, a reflection of endogenous insulin secretion, was 1.9 (IQR: $1.1-2.5$ ) $\mathrm{ng} / \mathrm{mL}$ at 90 days. The median exogenous insulin requirement was 0.4 (IQR: 0.3-0.6) unit/kg/day at 90 days, down from 0.5 (IQR: $0.4-0.8)$ unit $/ \mathrm{kg} /$ day at the time of discharge. This decrease in exogenous insulin requirements was statistically significant $(P=.02)$. One patient was no longer requiring exogenous insulin at 90 days.

\subsection{Health-related quality of life}

The SF-36 was completed by 11 patients preoperatively and at 90 days postoperatively. Physical health and total SF-36 scores were significantly improved at 90 days (Figure 3). Preoperatively, the median SF-36 physical health score was 34.0, and it improved to 54.6 at 90 days $(P=.008)$. Total SF-36 scores improved from a median of 47.1 to $65.3(P=.007)$. The median mental health score increased at 90 days but was not statistically significant $(P=.12)$. The SF-10 was completed by parents of 13 patients. The physical health score was significantly improved at 90 days postoperatively (13.4 vs $33.1, P=.01)$. The median psychosocial component also increased, but the increase was not statistically significant (43.6 vs $51.6, P=.38)$.

\section{4 | DISCUSSION}

Pediatric CP and ARP are debilitating diseases that can effectively be treated with TPIAT when significant pain and impaired quality of life persist despite maximal medical and endoscopic therapy. Patients are carefully selected, recognizing that the operative intervention itself has significant risk and short-term morbidity, but overall outcomes, both in-hospital and at 90 days postoperatively, remain excellent. This study is the first to demonstrate that pediatric patients with ARP/CP who undergo TPIAT have significant improvements in opioid and PN dependence at 90 days postoperatively. Stimulated $\mathrm{C}$-peptide levels are measurable at 90 days, demonstrating active $\beta$-cell function, and exogenous insulin requirements are significantly decreased at 90 days compared with the time of discharge. Importantly, HRQOL scores significantly improved in this population, as early as 3 months postoperatively.

While TPIAT has been performed in adults for 40 years, its introduction and broad implementation in children are significantly more recent. The pediatric population presents additional technical challenges with smaller bile ducts for reconstruction, as well as a theoretical increased risk of portal vein thrombus given smaller-caliber vasculature. However, surgical intervention in pediatric patients with $\mathrm{CP}$ who have had a shorter duration of disease relative to adult patients with CP may allow for isolation and autotransplant of islets before progressive destruction of the gland with advanced fibrosis, islet cell loss, and consequent endocrine insufficiency. Genetic risk factors are known to result in the development of $\mathrm{CP}$, including cationic trypsinogen gene (PRSS1), serine protease inhibitor

Am J Transplant. Author manuscript; available in PMC 2021 November 10. 
Kazal type 1 (SPINK1), cystic fibrosis transmembrane conductance regulator (CFTR), and chymotrypsin $\mathrm{C}($ CTRC) mutations, with $73 \%$ of children possessing at least 1 mutation in a pancreatitis-related gene in the INSPPIRE cohort. ${ }^{21,27,28}$ In these patients specifically, with the high likelihood of progressive $\mathrm{CP}$, the absence of anatomic characteristics that would allow surgical drainage procedures or local resections, and the life-time risk of pancreatic adenocarcinoma, ${ }^{29}$ early intervention in pediatric patients may have a significant long-term impact.

The benefits of TPIAT in pediatric patients include the ability to wean off of opioid medications as well as PN. ${ }^{30,31} \mathrm{PN}$ has significant risk and cost associated with it, including the risk of central line-associated bloodstream infections. ${ }^{32}$ However, at present, the overarching indication for TPIAT is for the treatment of chronic debilitating symptoms. Chronic pain has been associated with depression, anxiety, and decreased quality of life in pediatric populations. ${ }^{33,34}$ Our study confirms previous reports of significant improvement in quality of life, with our outcomes demonstrating significant improvement as early as 90 days postoperatively. Bellin et al, in their 2011 series of 19 consecutive pediatric patients undergoing TPIAT, found that patients had below average HRQOL before surgery based on SF-36, with significant improvement in the mean physical health score and a strong trend toward improvement in the mean mental health score at 1 year postoperatively. ${ }^{12}$ Wilson et al reported their experience with 14 adolescent patients who underwent TPIAT and found that SF-36 scores at 9 months were significantly increased compared with preoperative baseline scores for this population. ${ }^{30}$ Our study is the first to demonstrate that HRQOL scores, as measured with both SF-36 and SF-10, improved in as short a period as 90 days after surgery, indicating the profound social and quality of life benefit pediatric patients experience from the elimination of chronic pain.

In addition, we found that although the operation itself is a major undertaking, requiring reconstruction of the alimentary and biliary systems, in addition to splenectomy, cholecystectomy, and appendectomy, the postoperative complications have been minimal. One patient required reexploration for a bowel obstruction after the initial hospital course, and an additional patient required immediate reexploration for bleeding. Otherwise, 5 patients underwent percutaneous drain placement into a fluid collection identified on a computed tomography scan obtained for postoperative fevers but required no further intervention and did not develop clinically significant infection. These complication rates are consistent with previously reported pediatric TPIAT complication rates. ${ }^{30,31}$ Optimal outcomes of TPIAT require careful patient selection based on rigorous multidisciplinary evaluation with surgery, gastroenterology, endocrinology, pain management physicians, and psychologists.

Our study does have limitations. It is a retrospective review of a small cohort of pediatric patients undergoing TPIAT with short-term follow-up. However, even with this small sample size and 90-day follow-up, we have been able to demonstrate significant improvements in opioid and PN use as well as quality of life. The quality of life data were collected prospectively, both preoperatively and at 90 days, reducing recall bias inherent in asking patients about changes in their quality of life retrospectively. The SF-36, used in this study, has not been validated in pediatric populations. However, it has previously been used in 
these adolescent CP populations to evaluate for improvements in quality of life. ${ }^{12,30}$ Last, our sample size for this study was small, which may have affected our ability to detect statistical significance. For example, we did not find the amount of morphine equivalents used by patients taking opioids to be statistically different at 90 days compared with preoperatively, although the median decreased from 20 to 7.5 morphine equivalents per day, which may be a reflection of the small sample size. As the number of pediatric patients undergoing TPIAT continues to grow and the current post-TPIAT patients extend further out from their surgical intervention, we hope to be able to assess these outcomes with larger sample size, as well assessing their long-term postoperative outcomes.

$\mathrm{ARP}$ and $\mathrm{CP}$ are debilitating diseases that are increasing in frequency in the pediatric population and can be effectively treated with TPIAT, with significant improvements in opioid and PN use as well as quality of life. Recovery of $\beta$-cell function has a longer expected postoperative course, but even at 90 days, patients show evidence of islet function and a decrease in exogenous insulin needs. Improvement in all of these parameters—opioid, parenteral nutrition, and exogenous insulin use, as well as HRQOL—can be demonstrated over a short period of time postoperatively.

\section{ACKNOWLEDGMENTS}

This work was supported by funding from the Academic Research Committee at Cincinnati Children's Hospital Medical Center.

\section{Abbreviations:}

\section{ARP}

acute recurrent pancreatitis

\section{BMI}

body mass index

\section{CCHMC}

Cincinnati Children's Hospital Medical Center

CFTR

cystic fibrosis transmembrane conductance regulator

\section{CPA1}

carboxypeptidase A1

CP

chronic pancreatitis

CTRC

chymotrypsin $\mathrm{C}$

\section{ERCP}

endoscopic retrograde cholangiopancreatography 


\section{HRQOL}

health-related quality of life

ICU

intensive care unit

IEQ

islet equivalents

\section{INSPPIRE}

International Study Group of Pediatric Pancreatitis: In Search for a Cure

PN

parenteral nutrition

PRSS1

cationic trypsinogen gene

SF-10

Short Form 10-Item Health Survey

SF-36

Short Form 36-Item Health Survey

\section{SPINK1}

serine protease inhibitor Kazal type 1

\section{TPIAT}

total pancreatectomy with islet autotransplantation

\section{REFERENCES}

1. Yadav D, Timmons L, Benson JT, et al. Incidence, prevalence and survival of chronic pancreatitis: a population-based study. Am J Gastroenterol. 2011;106:2192-2199. [PubMed: 21946280]

2. Everhart JE, Ruhl CE. Burden of digestive diseases in the United States Part III: liver, biliary tract, and pancreas. Gastroenterology. 2009;136:1134-1144. [PubMed: 19245868]

3. Peery AF, Dellon ES, Lund J, et al. Burden of gastrointestinal disease in the United States: 2012 update. Gastroenterology. 2012;143:1179-1187. [PubMed: 22885331]

4. Keim V, Witt H, Bauer N, et al. The course of genetically determined chronic pancreatitis. JOP. 2003;4:146-154. [PubMed: 12853682]

5. Rebours V, Boutron-Ruault MC, Schnee M, et al. The natural history of hereditary pancreatitis: a national series. Gut. 2009;58:97-103. [PubMed: 18755888]

6. Morinville VD, Barmada MM, Lowe ME. Increasing incidence of acute pancreatitis at an American pediatric tertiary care center: is greater awareness among physicians responsible? Pancreas. 2010;39:5-8. [PubMed: 19752770]

7. Srinath AI, Lowe ME. Pediatric pancreatitis. Pediatr Rev. 2013;34:79-89. [PubMed: 23378615]

8. Abu-el-Haija M, El-Dika S, Hinton A, et al. Acute pancreatitis admission trends: a national estimate through the Kids' Inpatient Database. J Pediatr. 2018;194:147-151. [PubMed: 29174078]

9. Howes N, Lerch MM, Greenhalf W, et al. Clinical and genetic characteristics of hereditary pancreatitis in Europe. Clin Gastroenterol Hepatol. 2004;2:252-261. [PubMed: 15017610] 
10. Varni JW, Limbers CA, Burwinkle TM. Impaired health-related quality of life in children and adolescents with chronic conditions: a comparative analysis of 10 disease clusters and 33 disease categories/severities utilizing PedsQL 4.0 Generic Core Scales. Health Qual Outcomes. 2007;5:43.

11. Ingerski LM, Modi AC, Hood KK, et al. Health-related quality of life across pediatric chronic conditions. J Pediatr. 2010;156:639-644. [PubMed: 20117793]

12. Bellin MD, Freeman ML, Schwarzenberg SJ, et al. Quality of life improves for pediatric patients after total pancreatectomy and islet autotransplant for chronic pancreatitis. Clin Gastroenterol Hepatol. 2011;9:793-799. [PubMed: 21683160]

13. Dominguez-Munoz JE, Phillips M. Nutritional therapy in chronic pancreatitis. Gasteroenterol Clin North Am. 2018;47:95-106.

14. Sutherland DE, Matas AJ, Najarian JS. Pancreatic islet cell transplantation. Surg Clin North Am. 2007;87:1477-1501. [PubMed: 18053843]

15. Ahmad SA, Lowy AM, Wary CJ, et al. Factors associated with insulin and narcotic independence after islet autotransplantation in patients with severe chronic pancreatitis. J Am Coll Surg. 2005;201:680-687. [PubMed: 16256909]

16. Wahoff DC, Paplois BE, Najarian JS, et al. Autologous islet transplantation to prevent diabetes after pancreatic resection. Ann Surg. 1995;222:562-575. [PubMed: 7574935]

17. Behrman SW, Mulloy M. Total pancreatectomy for the treatment of chronic pancreatitis: indications, outcomes and recommendations. Am Surg. 2006;72:297-302. [PubMed: 16676850]

18. Chinnakotla S, Radosevich DM, Dunn TB, et al. Long term outcomes of total pancreatectomy and islet autotransplantation for hereditary/genetic pancreatitis. J Am Coll Surg. 2014;218:530-543. [PubMed: 24655839]

19. Wahoff DC, Paplois BE, Najarian JS, et al. Islet autotransplantation after total pancreatectomy in a child. J Pediatr Surg. 1996;31:132-135. [PubMed: 8632266]

20. Chinnakotla S, Bellin MD, Schwarzenberg SJ. Total pancreatectomy and islet autotransplantation in children for chronic pancreatitis. Ann Surg. 2014;260:56-64. [PubMed: 24509206]

21. Morinville VD, Hussain SZ, Bai H, et al. Definitions of pediatric pancreatitis and survey of current clinical practices: report from INSPPIRE (International Study Group of Pediatric Pancreatitis: in Search for a Cure). Pediatr Gastroenterol Nutr. 2012;55:261-265.

22. Ricordi C, Lacy PE, Finke EH, et al. Automated method for isolation of human pancreatic islets. Diabetes. 1988;37:413-420. [PubMed: 3288530]

23. Goto M, Johansson H, Maeda A, et al. Low molecular weight dextran sulfate prevents the instant blood-mediated inflammatory reaction induced by adult porcine islets. Transplantation. 2004;77:741-747. [PubMed: 15021838]

24. Greenbaum CJ, Mandrup-Poulsen T, McGee PF, et al. Mixed-meal tolerance test versus glucagon stimulation test for the assessment of beta-cell function in therapeutic trials in type 1 diabetes. Diabetes Care. 2008;31:1966-1971. [PubMed: 18628574]

25. Ware JE Jr, Sherbourne CD. The MOS 36-Item Short-Form Health Survey (SF-36). I. Conceptual Framework And Item Selection. Med Care. 1992;30:473-483. [PubMed: 1593914]

26. Saris-Baglama RN, DeRosa MA, Raczel AE, et al. The SF-10 Health Survey for Children: A User's Guide. Lincoln, RI: QualityMetric Incorporated; 2007.

27. Whitcomb DC. Hereditary pancreatitis: new insights into acute and chronic pancreatitis. Gut. 1999;45:317-322. [PubMed: 10446089]

28. Kumar S, Ooi CY, Werlin S, et al. Risk factors associated with pediatric acute recurrent and chronic pancreatitis: lessons from INSPPIRE. JAMA Pediatr. 2016;170:562-569. [PubMed: 27064572]

29. Rebours V, Boutron-Ruault M, Schnee M, et al. Risk of pancreatic adenocarcinoma in patients with hereditary pancreatitis: a national exhaustive series. Am J Gastroenterol. 2008;103:111-119. [PubMed: 18184119]

30. Wilson GC, Sutton JM, Salehi M, et al. Surgical outcomes after total pancreatectomy and islet cell autotransplantation in pediatric patients. Surgery. 2013;154:777-784. [PubMed: 24074415]

31. Bellin MD, Forlenza GP, Majumder K, et al. Total pancreatectomy with islet autotransplantation resolves pain in young children with severe chronic pancreatitis. JPGN. 2017;64:440-445. [PubMed: 28231072] 
32. Carter JH, Langley JM, Kuhle S, Kirkland S. Risk factors for central venous catheter-associated bloodstream infection in pediatric patients: a cohort study. Infect Control Hosp Epidemiol. 2016;37:939-945. [PubMed: 27139311]

33. Howard RF. Current status of pain management in children. JAMA. 2003;290:2464-2469. [PubMed: 14612483]

34. King S, Chambers CT, Huguet A, et al. The epidemiology of chronic pain in children and adolescents revisited: a systematic review. Pain. 2011;152:2729-2738. [PubMed: 22078064] 


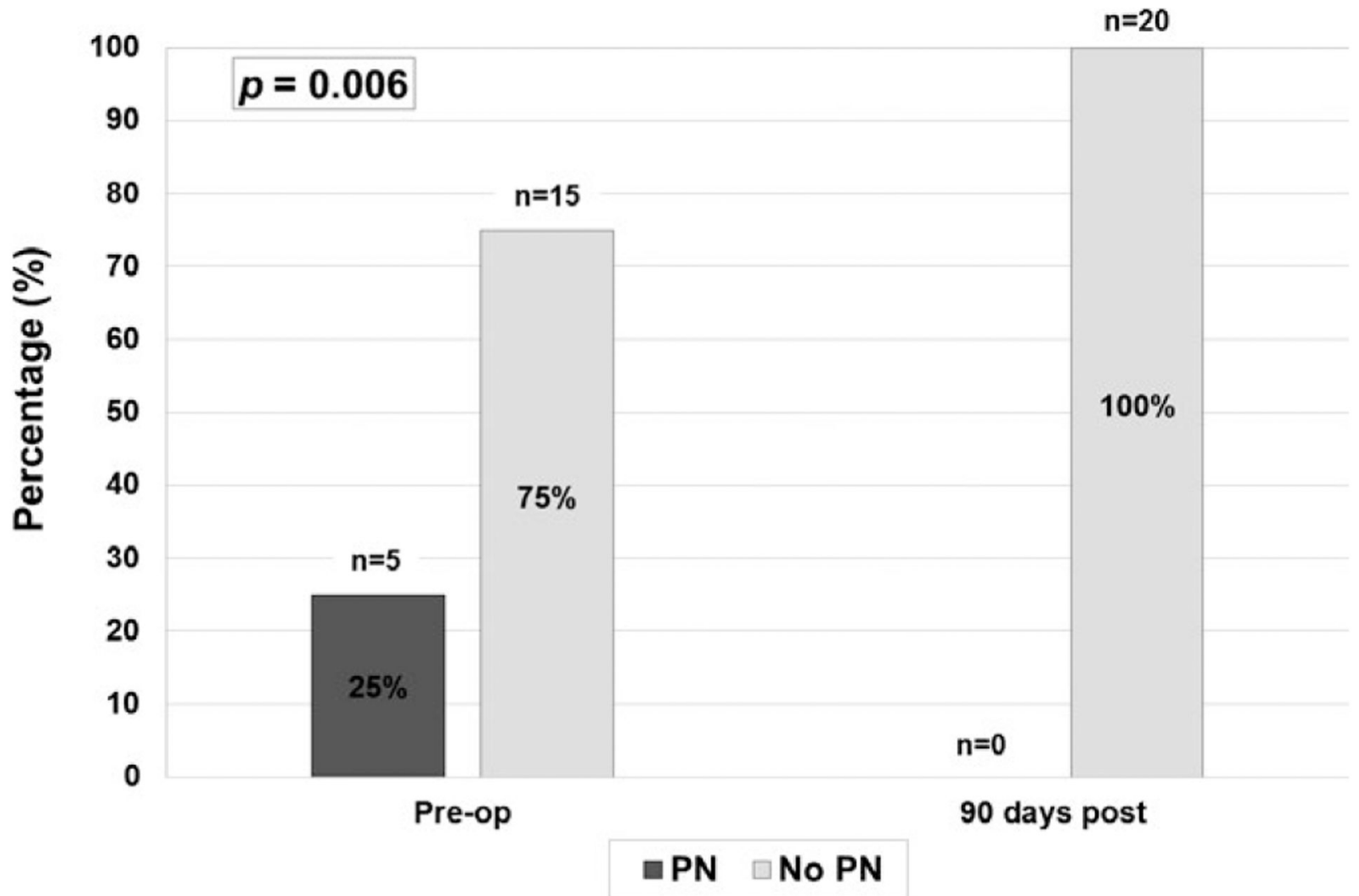

FIGURE 1.

Parenteral nutrition (PN) use preoperatively and postoperatively. This figure compares the percentage of patients requiring $\mathrm{PN}$ preoperatively with the percentage requiring PN postoperatively. By 90 days postoperatively, no patient required PN support $(P=.006)$ 
100

90

$$
p=0.01
$$

80

\section{$n=15$}

2 70

50

40

30

20

10

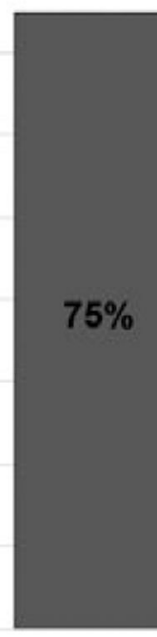

0

Pre-op

$n=5$

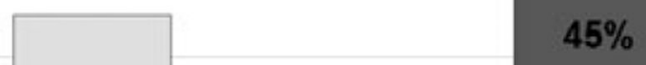

$25 \%$
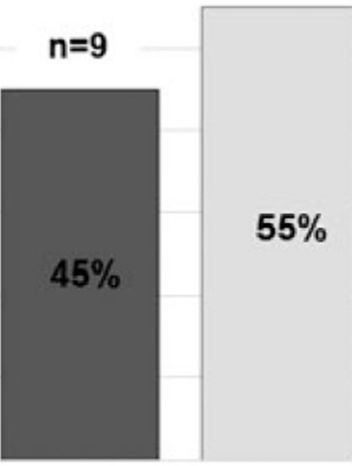

\section{0 days post}

\section{$\square$ On Opioids $\square$ Off Opioids}

FIGURE 2.

Opioid use preoperatively and postoperatively. This figure compares the percentage of patients on and off opioids preoperatively and at 90 days postoperatively, with a significant decrease in the proportion of patients requiring opioids at 90 days 


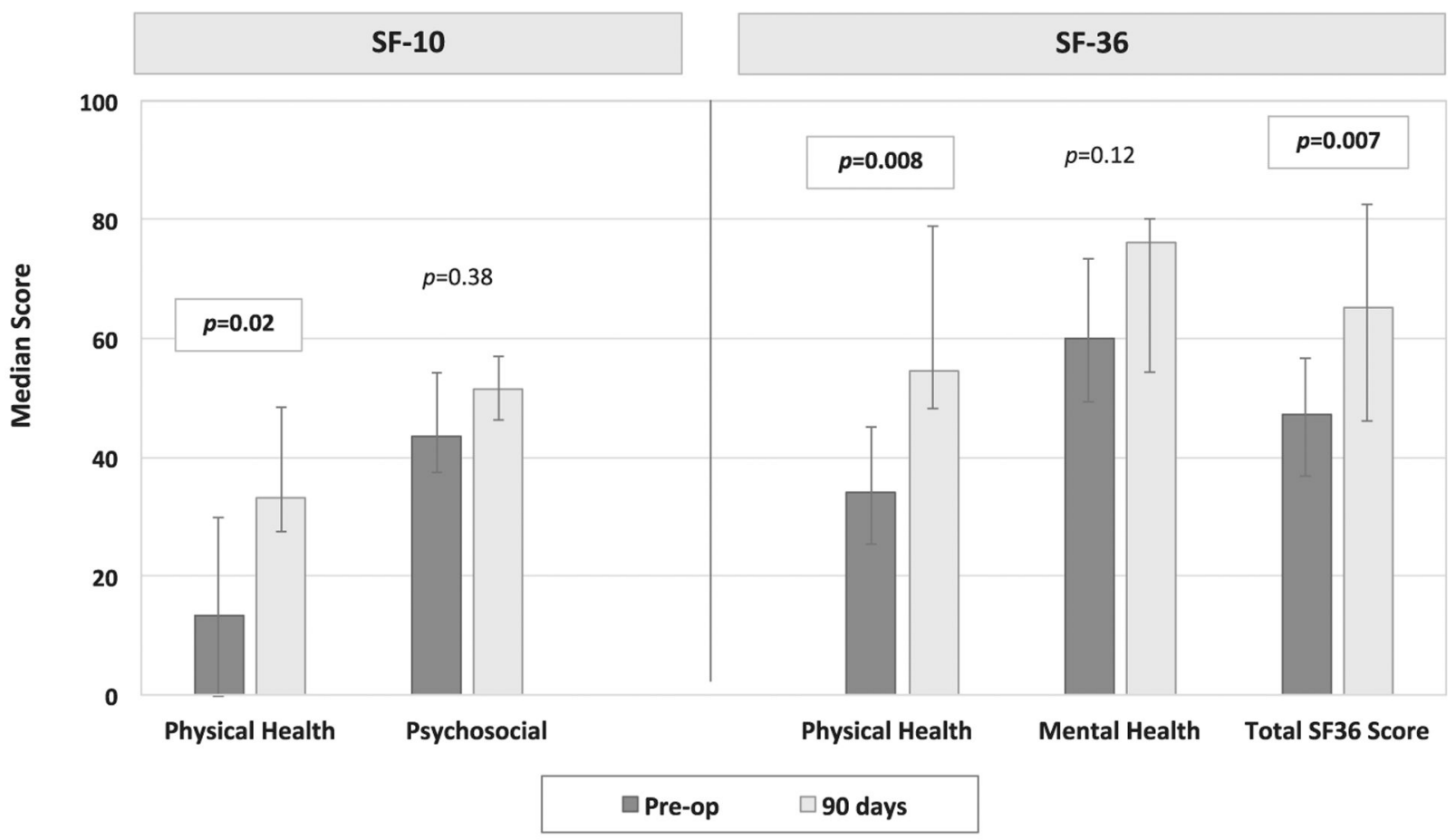

FIGURE 3.

Health-related quality of life preoperatively and postoperatively. This figure compares health-related quality of life scores in patients preoperatively and at 90 days postoperatively. The SF-10 was completed for patients $<12$ years of age. The SF-36 was completed for patients 12 years and older. The bars in the figure represent median scores, and the error bars demonstrate the IQR. SF-10 physical health scores and SF-36 physical health module scores and total scores significantly improved at 90 days postoperatively 


\section{TABLE 1}

Preoperative demographic and patient characteristics of children undergoing TPIAT

\begin{tabular}{|c|c|}
\hline Characteristic & No. of children $(\mathbf{N}=\mathbf{2 0})$ \\
\hline Age at TPIAT, $y$ & 13.5 (10.4 to 16.2$)$ \\
\hline \multicolumn{2}{|l|}{ Sex } \\
\hline Male & $7(35)$ \\
\hline Female & $13(65)$ \\
\hline BMI percentile & $84.8(63.8$ to 93.6$)$ \\
\hline Weight percentile & 81.5 (49.5 to 93.9$)$ \\
\hline \multicolumn{2}{|l|}{ Classification of pancreatitis } \\
\hline Chronic & $19(95)$ \\
\hline Acute recurrent alone & $1(5)$ \\
\hline \multicolumn{2}{|l|}{ Etiology of chronic pancreatitis } \\
\hline Genetic risk factor & $16(80)$ \\
\hline PRSS1 & $4(20)$ \\
\hline SPINK1 & $5(25)$ \\
\hline CFTR & $7(35)$ \\
\hline CTRC & $1(5)$ \\
\hline$C P A 1$ & $1(5)$ \\
\hline Anatomic association & $9(45)$ \\
\hline Pancreas divisum & $8(40)$ \\
\hline Annular pancreas & $1(5)$ \\
\hline No. of hospitalizations in preceding year & $4.5(3.5$ to 7.5$)$ \\
\hline No. of ERCPs in preceding year & $1.0(0.0$ to 2.0$)$ \\
\hline \multicolumn{2}{|l|}{ Preoperative opioid use in preceding year } \\
\hline Yes & $15(75)$ \\
\hline No & $5(25)$ \\
\hline \multicolumn{2}{|l|}{ Exocrine pancreatic insufficiency } \\
\hline Yes & $12(60)$ \\
\hline No & $6(30)$ \\
\hline Unknown & $2(10)$ \\
\hline \multicolumn{2}{|l|}{ Endocrine pancreatic dysfunction: diabetes } \\
\hline Yes & $3(15)$ \\
\hline No & $17(85)$ \\
\hline \multicolumn{2}{|l|}{ Preoperative nutrition } \\
\hline Total parenteral nutrition only & $4(20)$ \\
\hline Parenteral and oral feeds & $1(5)$ \\
\hline Enteral and oral feeds & $3(15)$ \\
\hline Oral diet only & $12(60)$ \\
\hline
\end{tabular}

Data presented as median (25th-75th percentiles) or $\mathrm{n}(\%)$.

BMI, body mass index; TPIAT, total pancreatectomy with islet autotransplantation; ERCP, endoscopic retrograde cholangiopancreatography. 
TABLE 2

Operative details and hospital stay

$\begin{array}{lc}\text { Operative details } & \\ \text { Operative duration, min } & 757.5(657.5 \text { to } 835.5) \\ \text { Islets } & 323100(226350 \text { to } 449400) \\ \quad \text { Total IEQ } & 6404(5018 \text { to } 7554) \\ \quad \text { Total lEQ/kg } & 17(85) \\ \text { Site of islet infusion } & 3(15) \\ \quad \text { Liver/intraportal only } & 10.0(8.5 \text { to } 11.0) \\ \quad \text { Liver/intraportal plus peritoneal cavity } & 26.5(22.5 \text { to } 31.0) \\ \text { ICU length of stay, days } & \\ \text { Overall length of stay, d } & \end{array}$

Data presented as median (25th-75th percentiles) or $\mathrm{n}(\%)$.

IEQ, islet equivalents, ICU, intensive care unit. 


\section{TABLE 3}

\section{Ninety-day outcomes}

\begin{tabular}{|c|c|}
\hline Outcome & No. of children $(N=20)$ \\
\hline \multicolumn{2}{|l|}{ Nutrition at 90 days } \\
\hline Any parenteral nutrition & 0 \\
\hline Enteral and oral feeds & $6(30)$ \\
\hline Oral diet only & $14(70)$ \\
\hline \multicolumn{2}{|l|}{ Opioid use at 90 days } \\
\hline Yes & $9(45)$ \\
\hline No & $11(55)$ \\
\hline \multicolumn{2}{|l|}{ Postoperative complications } \\
\hline Percutaneous drainage of fluid collection & $5(25)$ \\
\hline Reexploration for bleeding & $1(5)$ \\
\hline Bowel obstruction & $1(5)$ \\
\hline Time to cessation of opioid use, $\mathrm{wk}^{a}$ & $12.0(6.0$ to 20.0$), \mathrm{n}=11$ \\
\hline Stimulated C-peptide, ng/mL & $1.9(1.1$ to 2.5$), \mathrm{n}=18$ \\
\hline Insulin requirement, units $/ \mathrm{kg} / \mathrm{d}$ & $0.4(0.3$ to 0.6$)$ \\
\hline
\end{tabular}

